Articles

\title{
An Escherichia coli expression system for glutamyl endopeptidases optimized by complete suppression of auto-degradation
}

\author{
Toshio Ono $^{\mathrm{a}}$, Takayuki K. Nemoto ${ }^{\mathrm{a}, *}$, Yu Shimoyama ${ }^{\mathrm{b}}$, Shigenobu Kimura ${ }^{\mathrm{b}}$, \\ Yuko Ohara-Nemoto ${ }^{a}$ \\ ${ }^{\mathrm{a}}$ Department of Oral Molecular Biology, Course of Medical and Dental Sciences, Nagasaki University \\ Graduate School of Biomedical Sciences, Nagasaki 852-8588, Japan \\ ${ }^{\mathrm{b}}$ Department of Oral Microbiology, Iwate Medical University School of Dentistry, Morioka 020-8505, \\ Japan
}

Running title: V8 protease expression by prevention of auto-degradation

*Corresponding author.

Takayuki K. Nemoto, Department of Oral Molecular Biology, Course of Medical and Dental Sciences, Nagasaki University, 1-7-1 Sakamoto, Nagasaki 852-8588, Japan,

Fax : +81958197642

Tel : +81958197640

E-mail address: tnemoto@nagasaki-u.ac.jp (T. K. Nemoto) 


\begin{abstract}
V8 protease (GluV8), a member of the glutamyl endopeptidase I family, isolated from the V8 strain of Staphylococcus aureus, is widely utilized for proteome analysis because of its unique substrate specificity and resistance to detergents. We recently developed an Escherichia coli expression system for the production of GluV8 based on a technique that suppresses the auto-proteolysis: the use of the prosequence of its homolog (GluSE) from Staphylococcus epidermidis as a chimeric form or the introduction of 4 substitutions in the prosequence of GluV8. In the present study, we refined this technique through 5 amino acid substitutions within the prosequence of GluV8 for complete suppression of the auto-degradation. As a result, the recovery of GluV8 proform was enhanced to 20 fg/cell, which was comparable to the level of a constitutive inactive form of GluV8, indicating complete suppression of the auto-proteolysis. This mutated propeptide was also effective for the expression of the mature sequence of the glutamyl endopeptidase from Staphylococcus warneri. The recombinant proteins were successfully converted to their active forms through a common cleavage mechanism mediated by thermolysin in vitro. This strategy may shed light on the way for the expression of the proteases that were scarcely produced in E. coli.
\end{abstract}

Keywords: Auto-proteolysis; Glutamyl endopeptidase; Prosequence; Staphylococcus warneri; Staphylococcus epidermidis 
${ }^{1}$ Abbreviations used: GluV8, glutamyl endopeptidase I of Staphylococcus aureus; GluSE and GluSW, GluV8 homologues of S. epidermidis and S. warneri, respectively; CBB, Coomassie brilliant blue; mAb, monoclonal antibody; PAGE, polyacrylamide gel electrophoresis; SDS, sodium dodecyl sulfate. 


\section{Introduction}

Staphylococcus aureus produces extracellular proteases, which are regarded as important virulence factors. One of the classically defined endoproteases is a serine protease, GluV8 ${ }^{1}$, known also as V8 protease/Glu-C/SspA [1]. An S. aureus GluV8-deficient mutant was severely attenuated in virulence in mouse abscess, bacteremia, and wound infection models [2]. S. aureus proteases modulate bacterial adhesive molecules, changing the phenotype from adhesive to invasive, and degrade fibronectin-binding proteins and protein A [2], which are adhesion molecules on the staphylococcal surface [3].

GluV8 belongs to the glutamyl endopeptidase I (EC 3.4.21.19) family and specifically cleaves the peptide bond after negatively charged residues, glutamic acid, and less potently, aspartic acid [4]. The nucleotide sequence encodes a protein of 336 amino acids that includes a prepropeptide consisting of 68 residues $\left(\mathrm{Met}_{1}-\mathrm{Asn}_{68}\right)$ and a C-terminal tail of 52 residues containing a 12-fold repeat of the tripeptide Pro-Asp/Asn-Asn [5]. Drapeau [6] reported that activation of the GluV8 precursor is achieved by a neutral metalloprotease. Shaw et al. [7] further demonstrated that the cascade reactions in processing of major extracellular pathogenic proteases of S. aureus from metalloprotease/aureolysin, GluV8/SspA, and finally, to cysteine protease/SspB.

We previously purified [8] and cloned [9] the glutamyl endopeptidase from Staphylococcus epidermidis (designated GluSE), which consists of 282 amino acids composed of a preprosequence (Met $\left.{ }_{1}-\mathrm{Ser}_{66}\right)$ and mature portion $\left(\mathrm{Val}_{67}-\mathrm{Gln}_{282}\right)$. GluSE does not have the C-terminal repeat sequence found in GluV8 [9]. Another Staphylococcal homolog, glutamyl endopeptidase from Staphylococcus warneri (designated GluSW), was cloned and deduced to be composed of 316 amino acids and to possess a 34-amino acid Asp/Asn-rich region at its C-terminus [10]. When GluV8 with its C-terminal repeat deleted was expressed in E. coli, it could fold to an active form after denaturation-renaturation 
treatment [11]. Thus, the C-terminal repeat sequence is not needed for the enzymatic activity and its role remains unknown. The presequence of GluV8 $\left(\mathrm{Met}_{1}-\mathrm{Ala}_{29}\right)$ functions as a signal peptide required for the secretion, and the prosequence $\left(\mathrm{Leu}_{30}-\mathrm{Asn}_{68}\right)$ for the suppression of the protease activity [12, 13]. A recent study proposed the sequential processing within the prosequence of GluV8 [12].

Expression of recombinant GluV8 in Escherichia coli would be useful to elucidate in detail the roles of the prepro- and C-terminal repeated segments as well as the specific amino acid residues involved in the processing and enzymatic activity. For instance, we have recently demonstrated the importance of the N-terminal $\mathrm{Val}_{69}$ and $\mathrm{Ser}_{237}$ by in vitro mutagenesis studies [13]. Because secreted bacterial proteases including GluV8 are neither membrane-bound nor glycosylated, an E. coli expression system should be suitable for their expression.

There are 2 major problems that could occur on the E. coli expression of proteases. At first, the expression of a target protein occasionally produces insoluble materials called inclusion bodies. Prior to our recent studies [13, 14], one E. coli expression study was reported [11], in which mature GluV8 was expressed as a fusion protein tagged to exogenous peptides at $\mathrm{N}$ - and $\mathrm{C}$-termini for prevention of its auto-activation. This form was expressed and purified as inclusion bodies. The mature protein was then obtained by the cleavage of the exogenous peptides, denaturation-renaturation, and then ion-exchange chromatography for purification. Because this procedure is time-consuming and the recovery at the denaturation-renaturation step is low (20\%), this is not generally employed for the purification. The commercially available GluV8 is still purified from the culture supernatant of the $S$. aureus V8 strain.

As a second problem, even if a target protein is expressed as a soluble form, the protease might be expressed or converted to a mature form in host cells. In particular, if the maturation occurs through 
an auto-proteolytic pathway, the expression of a target protease may be toxic for host cells. Accordingly, one of the ideal expression systems should be the expression of a soluble and latent form of a protease in E. coli and the subsequent in vitro maturation processing.

We recently developed an expression system for GluSE in E. coli $[13,14]$. GluSE was expressed as a full-length form with a His hexamer tag at its C-terminus. The purified GluSE was recovered as enzymatically inactive proforms, which contained the full or parts of its prosegment. In contrast, GluV8 was poorly expressed by the identical system due to auto-degradation. The comparison of their prosequences suggested auto-proteolytic degradations of the GluV8 prosequence at the $\mathrm{Glu}_{62}-\mathrm{Lys}_{63}$ and $\mathrm{Glu}_{65}-\mathrm{Arg}_{66}$ bonds (Fig. 1a). In fact, GluV8 was efficiently recovered by swapping its preprosegment with that of GluSE or by the introduction of 4 amino acid substitutions to the prosequence of GluV8 [13]. However, the expression level of GluV8 was still lower than that of GluSE. Considering the low similarity of the prosequences between GluSE and GluV8, we speculated that the prepropeptide of GluSE can not completely compensate the role of that of GluV8 and that the 4 substitutions did not fully suppress the auto-degradation.

In the present study, to evaluate the auto-degradation occurring during the expression, we utilized a constitutively inactive form of GluV8, the active $\operatorname{Ser}_{237}$ of which was substituted to Ala [13]. By use of this form as a positive control, we established an optimal expression system that completely suppressed the auto-degradation of GluV8 in E. coli. We also tried to express another member of the family proteases, GluSW, which possessed 3 Glu and 2 Asp within the prosequence. The identification of the N-terminus of its mature form would be helpful for the understanding of the functioning mechanism of GluSW. 


\section{Materials and methods}

\section{Materials}

The materials used and their sources were the following: Expression vector pQE60 and plasmid pREP4, from Qiagen Inc. (Chatsworth, CA); low-molecular-weight markers, from GE Healthcare (Buchinghamshire, England); restriction enzymes and DNA-modifying enzymes, from Nippon Gene (Tokyo, Japan); KOD plus DNA polymerase, from Toyobo (Tokyo, Japan); Z-Leu-Leu-Glu-MCA and other MCA peptides, from Peptide Institute Inc. (Osaka, Japan); azocasein, from Sigma; Talon metal affinity resin (His ${ }_{6}$-protein binding capacity, $5.3 \mathrm{mg} / \mathrm{ml}$ ), from Clontech Laboratories Inc. (Palo Alto, CA); anti-penta-His monoclonal antibody (mAb), from Qiagen Inc.; and alkaline-phosphatase-conjugated rabbit anti-mouse $\operatorname{Ig}(\mathrm{G}+\mathrm{A}+\mathrm{M})$, from Zymed Laboratories Inc. (San Francisco, CA, USA). Oligonucleotide primers were purchased from Genenet (Fukuoka, Japan).

Bacterial expression vectors

All recombinant proteins were expressed in E. coli with a His hexamer tag at their C-terminus by use of the pQE60 expression vector. The constructs and their modified positions are summarized (Table 1).

Genomic DNA was purified from S. warneri JCM 2415 as described previously [15]. The DNA fragment encoding the full-length form of GluSW was amplified by PCR with a pair of primers (sense 5'-TTTGGATCCAAAGTTAAGTTTTTACAGCA and anti-sense, 5’-GGCGGATCCCGCTGCGTCTGGATTATCGTA). BamHI sites are indicated by italic letters. The resultant PCR fragment was inserted into a BamHI site of pQE60 after being cleaved with BamHI. Y1090[pREP4] cells were transformed with the plasmid. Expressions of GluSE, GluV8, a chimeric 
protein of prepro-GluSE and mature GluV8, GluV8 with 2 (GluV8mut2) or 4 (GluV8mut4) amino acid substitutions, and GluSE and GluV8 with the substitution at their active Ser were described previously $[13,14]$

The plasmids encoding GluV8 with the 5 amino acid substitutions (designated GluV8mut5) and GluV8mut5 with its C-terminal repeat deleted (designated GluV8mut5 $\Delta$ C) were prepared by PCR with pQE60-GluV8mut4 and GluV8mut4 $4 \mathrm{C}$ as templates with sense (5’-AATCATCCACAACAAACGCAGTCAAG) and anti-sense (5'-GTGCATAGCCTTTGAAGATAACGCGTT) primers. A mutated nucleotide (C>G) responsible for the substitution from $\mathrm{Asp}_{36}$ to His is underlined. PCR was performed for 30 cycles by use of the KOD plus system, which did not add any nucleotide at the 3'-OH end of PCR fragments. After incubation with DpnI for degradation of the template, the PCR product was purified. Thereafter, the 5'-end of the PCR fragment was phosphorylated by T4 polynucleotide kinase, and then, the phosphorylated fragment was self-ligated by T4 DNA ligase in one tube simultaneously. Y1090[pREP4] cells were transformed with the resulting plasmid.

The plasmid encoding prepro-GluV8 with the 5-amino acid substitutions and mature GluSW (designated GluV8mut5-SW) was prepared by PCR with a pair of primers (sense, 5'-GTCATACTACCTAACAATGATCGACATCAA; and anti-sense, 5'-ACTTGGGTGACTACGTTGTTGTAATGGTTT). The former targeted the sequence of mature GluSW and the latter the preprosequence of GluV8mut5. A mixture of pQE60-GluV8mut5 and pQE60-GluSW was used as template. The resultant 5-kb fragment was self-ligated and Y1090[pREP4] was transformed with the resultant plasmid as described above. The mutations of nucleotides were confirmed by DNA sequencing. 


\section{Expression and purification of recombinant proteins}

Recombinant proteins were expressed and purified as described previously [16]. Briefly, E. coli Y1090[pREP4] (300 ml) carrying pQE60-derived expression plasmids was cultured overnight at $37^{\circ} \mathrm{C}$ in LB broth containing $50 \mu \mathrm{g} / \mathrm{ml}$ of ampicillin and $25 \mu \mathrm{g} / \mathrm{ml}$ of kanamycin. Protein expression was induced by dilution of the culture with LB broth $(500 \mathrm{ml})$ containing $0.2 \mathrm{mM}$ isopropyl $\beta$-D-thiogalactopyranoside and $50 \mu \mathrm{g} / \mathrm{ml}$ of ampicillin and incubation at $30^{\circ} \mathrm{C}$ for $3 \mathrm{~h}$. Bacterial cells were harvested by centrifugation and lysed with $50 \mathrm{ml}$ of lysis/washing buffer (20 mM Tris-HCl, pH 8.0, containing $0.1 \mathrm{M} \mathrm{NaCl}$ and $10 \mathrm{mM}$ imidazole) to which $0.5 \mathrm{mg} / \mathrm{ml}$ of lysozyme and $10 \mu \mathrm{g} / \mathrm{ml}$ of leupeptin had been added. Recombinant proteins were recovered in the cell lysate fraction and then purified by an affinity chromatography $(1 \times 5 \mathrm{~cm}$ ) with Talon metal affinity resin according to the manufacturer's protocol, except that $10 \mathrm{mM}$ imidazole was included in the lysis/washing buffer. After washing of unbound materials, bound proteins ( $1 \mathrm{ml} /$ fraction) were eluted with $0.1 \mathrm{M}$ imidazole $(\mathrm{pH}$ 8.0) containing $10 \%(\mathrm{v} / \mathrm{v})$ glycerol. Purified proteins were stored at $-80^{\circ} \mathrm{C}$ until used.

For comparison in the expression level of 12 recombinant proteins (Fig. 2), they were expressed as described above in the following volumes: An overnight culture (3 ml) of cells was diluted to $10 \mathrm{ml}$ for induction. After harvest, the cells were lysed with $1 \mathrm{ml}$ of the lysis solution and the lysate $(1 \mathrm{ml})$ was adsorbed onto a Talon affinity resin (20 $\mu$ l of bed volume) for 5 min. After extensive washing of the unbound materials, $40 \mu \mathrm{l}$ of SDS-buffer was added. The resin was then heated to denature bound proteins. Thereafter, aliquots $(5 \mu \mathrm{l})$ of the lysates or bound proteins were separated by SDS-PAGE. The intensity of CBB-stained bands of purified proteins was quantified by use of PDQuest 2-D Analysis Software (Bio-Rad). 
In vitro processing and the measurement of protease activity

Unless otherwise stated, the in vitro processing of recombinant proteins and subsequent protease assay were performed as follows: Recombinant proteins $(3 \mu \mathrm{M})$ were incubated in $0.1 \mathrm{ml}$ of $10 \mathrm{mM}$ sodium borate, $\mathrm{pH}$ 8.0, 0.005\% (v/v) Triton X100 containing $2 \mathrm{mM} \mathrm{CaCl}_{2}$ with 2.8 (ratio 110:1) or 8.4 pmol (ratio 37:1) of thermolysin at $37^{\circ} \mathrm{C}$ for $4 \mathrm{~h}$. Thereafter, aliquots of glutamic acid-specific proteases (40 nM) were incubated with $20 \mu \mathrm{M}$ Z-Leu-Leu-Glu-MCA in $0.2 \mathrm{ml}$ of $50 \mathrm{mM}$ Tris-HCl (pH 8.0) and $5 \mathrm{mM}$ EDTA at $25^{\circ} \mathrm{C}$ for $2 \mathrm{~h}$. The fluorescence was measured with an excitation at $380 \mathrm{~nm}$ and emission at $460 \mathrm{~nm}$ with a Fluorescence Photometer F-4000 (Hitachi, Tokyo, Japan).

Protease activity was also determined by colorimetric measurement by use of azocasein (Sigma). Following the incubation of the proteases $(57 \mathrm{pmol} / 75 \mu \mathrm{l})$ with $2 \mathrm{mg} / \mathrm{ml}$ of azocasein in $50 \mathrm{mM}$ Tris- $\mathrm{HCl}\left(\mathrm{pH}\right.$ 8.0) containing $5 \mathrm{mM}$ EDTA at $37^{\circ} \mathrm{C}$ for $1 \mathrm{~h}$, the reaction was stopped by the addition of $15 \%(\mathrm{w} / \mathrm{v})$ ice-cold trichloroacetic acid at a final concentration of $4 \%(\mathrm{w} / \mathrm{v})$. After a 10-min incubation at $0^{\circ} \mathrm{C}$, the supernatant obtained by centrifugation at $10,000 \mathrm{x} g$ for 5 min was mixed with an equal volume of $0.5 \mathrm{M} \mathrm{NaOH}$, and then the absorbance at $440 \mathrm{~nm}$ was measured.

Sodium dodecylsulfate (SDS)-polyacrylamide gel electrophoresis (PAGE)

Purified proteins $(0.8 \mu \mathrm{g})$ were separated by PAGE in the presence of $0.1 \%$ SDS at a polyacrylamide concentration of $12 \%(w / v)$. In the experiments of Figs. 2 and 5, aliquots (5 $\mu \mathrm{l})$ of the lysates or purified fractions were loaded onto an SDS-PAGE gel. Separated proteins were stained with Coomassie Brilliant Blue (CBB) or subjected to the immunoblotting with anti-penta His mAb (0.2 $\mu \mathrm{g} / \mathrm{ml})$ as described previously [13]. 
$N$-terminal amino acid sequencing

N-terminal amino acid sequences were determined after separation of recombinant proteins by SDS-PAGE and transference to a polyvinylidene difluoride membrane (Sequi-Blot PVDF Membrane,

Bio-Rad). After having been stained with CBB, the bands were excised and directly sequenced with a model Precise 49XcLC protein sequencer (ABI).

Protein Concentration

Protein concentrations were determined by the bicinchoninic acid method (Pierce). Bovine serum albumin dissolved in $0.1 \mathrm{M}$ imidazole $(\mathrm{pH} 8)$ and $10 \%(\mathrm{v} / \mathrm{v})$ glycerol was used as a reference to adjust buffer compositions to affinity-purified samples. 


\section{Results}

Comparison of the expression levels among the glutamyl endopeptidases in E. coli

Comparison of the amino acid sequences of the GluSE, GluV8, and GluSW except for the C-terminally extra regions revealed the highest homology at the mature sequence (58.8 to $80.1 \%$ ), followed by the presequence (29.6 to 55.2\%), and lowest at the prosequence (15.4 to 51.3\%) (Fig. 1). Among them, the mature sequences are the most conserved between GluV8 and GluSW (80.1\%), indicating their close kinship. The homology of the prosequence is also highest between GluV8 and GluSW (51.3\%).

GluSE, GluV8, GluSW, and their derivatives (Table 1) were expressed in E. coli with a C-terminal His hexamer tag. The immunoblotting analysis of the lysates revealed that all of the recombinant proteins had been produced (Fig. 2a). The appearance of several bands in most lanes indicated their partial degradation. CBB-staining of bacterial lysate proteins revealed 32-kDa (Fig. 2a, lanes 1, 7, 8, and 10) and 38-kDa (lane 12) bands distinguished from those of endogenous bacterial proteins.

In order to quantify the expression, we performed batch purification with $1 \mathrm{ml}$ of the lysate (Fig. 2b). The intensity of the purified bands may be considered to reflect their expression levels. GluSE was expressed efficiently (Fig. 2b, lane 1), whereas GluV8 was scarcely expressed (lane 2). When the chimeric protein GluSE-V8, i.e., the prepro- and mature sequences of which were derived from GluSE and GluV8, respectively, was expressed, the density of the 44-kDa band was increased, indicating the suppression of degradation (lane 3).

Enhancement of the expression of recombinant GluV8 by modification of the prosegment

Instead of using the prepropeptide of GluSE, we serially substituted in the GluV8 prepropeptide 
several amino acids constituting the peptide bonds potentially auto-proteolysed to those located at the equivalent positions in GluSE (Fig. 1a). At first, 2 amino acid substitutions, i.e., from Glu ${ }_{62}$ and Glu 65 to $\operatorname{Gln}_{62}$ and $\operatorname{Ser}_{65}$ (GluV8mut2) partially increased the density of the 39-44 kDa bands (Fig. 2b, lane 4) compared with the density of the bands of GluV8 possessing its authentic prepropeptide (lane 2). When 2 additional amino acids, i.e., $\mathrm{Ala}_{67}$ and $\mathrm{Asn}_{68}$, were substituted to $\mathrm{Pro}_{67}$ and $\mathrm{Ser}_{68}$ (GluV8mut4), the 44-kDa band density increased (Fig. 2b, lane 5). We further expressed GluV8mut5, which carried an additional substitution of $\mathrm{Asp}_{36}$ to His. GluV8mut5 (Fig. 2b, lane 6) showed a 44-kDa band having much higher density than that of GluV8mut4 (lane 5). Concomitantly, the degradation products smaller than 44 kDa of GluV8 and GluV8mut2 (Fig. 2a, lower panel, lanes 2 and 4) disappeared on GluV8mut4 and GluV8mut5 (lanes 5 and 6). We postulated that the deletion of the C-terminal repeat sequence might further enhance the expression level of GluV8, because a shorter polypeptide might be more efficiently translated in E. coli. In fact, the expression level of GluV8mut5 $\Delta \mathrm{C}$ was additionally increased (Fig. 2b, lane 7).

\section{Expression of the constitutively inactive form of GluSE and GluV8}

It remained unknown whether the auto-proteolysis was completely suppressed by the 5 substitutions or further suppression was possible. To settle this issue, we compared the expression level of that molecule to that of the constitutively inactive form, the active Ser residue of which was substituted to Ala, because the expression level of the inactive form would be expected to be maximal. In fact, the constitutively inactive forms of GluSE and GluV8 were abundantly expressed in E. coli (Fig. 2, lanes 8-10). The band densities of GluV8mut5 and GluV8mut5 $\Delta \mathrm{C}$ (lanes 6 and 7) were comparable to those of GluV8mut4 Ser237Ala and GluV8mut4 $\Delta$ C Ser237Ala (lanes 9 and 10), respectively. 
Taken together, these results indicate that the auto-degradation was completely suppressed in GluV8mut5 and GluV8mut5 $\Delta$ C.

\section{Expression of GluSW}

The E. coli expression system of GluV8 developed herein may be applicable to the expression of other members. As an initial attempt, we applied this technique to the glutamyl endopeptidase from $S$. warneri, GluSW. The full-length form of GluSW was recovered as 35-, 38- and 40-kDa species (Fig. 2b, lane 11), indicating proteolytic degradation. Because there are Glu residues at positions 28, 60, and 63 and Asp ones at positions 34 and 51, the substitutions of the 5 amino acids to non-auto-cleavable ones were postulated to enhance its expression level. However, we here employed another strategy: The amino acid sequence at the GluSW proregion is $51.3 \%$ identical to the corresponding region of GluV8. Therefore, we suspected that the prosequence of GluV8mut5 might compensate this role. In fact, a chimeric protein carrying the mutated preprosequence of GluV8 and mature sequence of GluSW (designated as GluV8mut5-SW) was efficiently expressed in E. coli as a 38-kDa species (Fig. 2, lane 12).

Large-scale expression of GluV8mut5 $4 \mathrm{C}$ and GluV8mut5-SW in E. coli

When the wild-type GluV8 and GluV8mut5 $\Delta \mathrm{C}$ were expressed in 800-ml cultures, the expression of GluV8mut5 $\Delta \mathrm{C}$ was much higher than that of GluV8 (Fig. 3a). Similarly, the expression of GluV8mut5-SW was much higher than that of the wild-type GluSW (Fig. 3b). Approximately, $20 \mathrm{fg}$

of recombinant GluV8mut5 $\Delta \mathrm{C}$ and $30 \mathrm{fg}$ of GluV8mut5-SW were purified per an E. coli Y1090[pREP4] cell. 
In vitro processing of the proteases by thermolysin

It has been reported that GluV8 is processed by aureolysin, a thermolysin-family metalloprotease [6, 7]. This processing was faithfully reproduced by thermolysin treatment of the recombinant proteins in vitro (Fig. 4a). The proteolytic activity toward Z-Leu-Leu-Glu-MCA was highest for GluV8 (Fig. 4b). The activity of GluSW was slightly higher than that of GluSE (Fig. 4c). The extremely high activity of GluV8, however, was dependent on substrates, because the proteolytic activity among the proteases toward azocasein showed only a 1.7-fold difference in range (Fig. 4d). Nevertheless, it should be noted that the order of the protease activity of the 3 proteases toward azocasein was consistent with their order toward Z-Leu-Leu-Glu-MCA.

\section{Identification of the N-terminus of mature GluSW}

Various polypeptides maintaining truncated prosequences were transiently produced during the processing of pro-glutamyl endopeptidases and finally, mature forms with the N-termini of $\mathrm{Val}_{67}$ for GluSE [14], $\mathrm{Val}_{69}$ for GluV8 [12, 13], and their equivalent amino acid, $\mathrm{Leu}_{48}$, for the protease from Enterococcus faecalris [17] were produced. In contrast, the 31-kDa mature form of GluSW was reported to retain $\operatorname{Arg}_{64}$-Ala-Asn 66 at its $\mathrm{N}$-terminus [10], which is equivalent to the C-terminal tripeptide, His $_{66}$-Ala-Asn 68 , of the GluV8 prosequence (Fig. 1a). If this form were truly the mature enzyme, GluSW may be activated by the auto-proteolytic cleavage at the $\mathrm{Glu}_{63}-\mathrm{Arg}_{64}$ bond (Fig. 1a, an arrow with X). Nevertheless, referred to our recent findings on recombinant GluV8 and GluSE [13, 14], it was speculated that the $\mathrm{Asn}_{66}-\mathrm{Val}_{67}$ bond is the true maturation processing site for GluSW. To settle these alternatives, we determined the proteolytic activity and the $\mathrm{N}$-terminal sequence of 
recombinant GluSW before and after thermolysin treatment. The 35-kDa wild type GluSW and 38-kDa GluV8mut5-SW possessed no proteolytic activity and the 33-kDa form produced by thermolysin treatment exerted the proteolytic activity (Fig. 5). The N-terminal sequence of the 35-kDa wild-type GluSW was Arg $_{64}$-Ala-Asn-Val-Ile-Leu-Pro-Asn-Asn ${ }_{72}$, indicating auto-degradation at the $\mathrm{Glu}_{63}-\mathrm{Arg}_{64}$ bond. In contrast, the N-terminal sequence of the 38-kDa GluV8mut5-SW was $\mathrm{Leu}_{30}$-Ser-Ser-Lys-Ala-Met-His-Gln ${ }_{37}$, demonstrating the proper processing at the pre-pro border (Fig. 1a). The N-terminal sequence of the enzymatically-active 33-kDa form of wild-type GluSW started from $\mathrm{Val}_{67}$. Taken together, these data indicate that the N-terminus of mature GluSW was not $\operatorname{Arg}_{64}$, but $\mathrm{Val}_{67}$.

Mechanism of the amino acid substitutions responsible for the enhanced expression of GluV8

In addition to the conversion of acidic amino acids to other ones, the replacement of $\mathrm{Ala}_{67}-\mathrm{Asn}_{68}$ to Pro-Ser contributed to the stabilization of GluV8 (Fig. 2, lanes 3 and 4). Why did this replacement enhance the recovery? We hypothesized that the processing site, $\mathrm{Asn}_{68}$ - $\mathrm{Val}_{69}$, could be partially cleaved by GluV8, because the residual group of Asn is relevant to that of Asp. To confirm this hypothesis, we compared the proteolytic activity of MCA peptides carrying various amino acids at their P1 position. The proteolytic activity of GluV8 was highest for the 2 peptide substrates carrying Glu at the P1 position and was significantly lower toward the 2 substrates carrying Asp at it (Table 3). Interestingly, GluV8 also possessed trace activity toward Z-Ala-Ala-Asn-MCA, which was at least 30-fold higher than those for residual MCA peptides that carried Ala, Phe, or Leu at their P1 position. Furthermore, even unprocessed GluV8 possessed a proteolytic activity toward Ac-Ala-Ala-Asn-MCA. At last, it should be noted that the C-terminal tri-peptide of the prosequence of GluV8, i.e., 
His $_{66}$-Ala-Asn 68 , resembles the unprocessed-GluV8-cleavable substrate, Ac-Ala-Ala-Asn-MCA.

\section{Discussion}

We recently developed an E. coli expression system for GluV8 either by swapping the propeptide of it to that of GluSE or 4-amino acid substitutions from the amino acids of GluV8 to those of GluSE at equivalent positions, i.e., Glu62Gln, Glu65Ser, Ala67Pro, and Asn68Ser [13]. The present study demonstrated that the fifth substitution, i.e., $\mathrm{Asp}_{36}$ of the propeptide to His, further enhanced the recovery of GluV8. This finding was surprising, because (i) the degradation activity of GluV8 for the Asp-X bond is substantially lower than that for the Glu-X bond (Table 3) [18], and (ii) the position is located near the pre-pro border $\left(\mathrm{Ala}_{29}-\mathrm{Leu}_{30}\right)$ of GluV8; thereby, even if the $\mathrm{Asp}_{36}-\mathrm{Asn}_{37}$ bond was hydrolyzed, the majority (82\%) of the prosequence was still tagged to the mature moiety, and this form was enzymatically inactive [13]. However, this mutation actually improved the recovery, suggesting that the whole sequence of the propeptide is needed for the maintenance of the GluV8 proform. It has been reported that the prosequence of subtilisin is an intrinsically unstructured polypeptide that adopts an $\alpha-\beta$ structure only in the presence of the mature protease [19]. If this was also the case for GluV8, the deletion of a short segment from the prosequence may have a profound effect on its structure.

The substitutions of $\mathrm{Ala}_{67}-\mathrm{Asn}_{68}$ to Pro-Ser additionally contributed to the enhancement of the expression of the recombinant protease. The proteolytic activity of non-processed GluV8 toward Z-Ala-Ala-Asn-MCA accounted 56\% of that of processed GluV8. This finding indicates that the non-processed GluV8 could cleave the $\mathrm{Asn}_{68}-\mathrm{Val}_{69}$ bond to some extent. It has been proposed that the $\alpha$-amino group of the $\mathrm{N}$-terminal residue is essential for the specific interaction with the $\gamma$-carboxyl 
group of Glu in a substrate peptide [20]. However, the protonation of the $\alpha$-carboxyl group does not take an advantage in the association with amide group of Asn at the P1 position. This would explain the reason why the Asn-X bond was hydrolyzed either by the unprocessed or processed GluV8.

The P2-position amino acid of the maturation processing site of GluV8 was substituted from $\mathrm{Ala}_{67}$ to Pro together with substitution of the P1 site from $\mathrm{Asn}_{68}$ to Ser. It has been reported that Phe at P2 position was preferable for the GluV8-family endopeptidases and that Ala and Pro at P2 position were not [18]. Therefore, the substitution from $\mathrm{Ala}_{67}$ to Pro may not affect the processing at the $\operatorname{Ser}_{68}-\mathrm{Val}_{69}$ bond of GluV8. Nevertheless, we should point out that the X-Pro and Pro-X bonds are highly resistant to most proteases including GluV8, which might additively contribute to the prevention of proteolytic degradation, as GluV8 with short prosequences could exert a residual activity [13].

The strategy developed for the expression of GluV8 in E. coli was successfully applied to the expression of another member of this family, GluSW. The N-terminal sequence of native GluSW was determined to $\mathrm{Arg}_{64}-\mathrm{Ala}-\mathrm{Asn}-\mathrm{Val}_{67}$, and this form is known to exert caseinolytic and glutamic acid-specific hydrolytic activities [10]. However, the present study clearly demonstrated that GluSW starting at $\operatorname{Arg}_{64}$ had no proteolytic activity and that the hydrolysis of the $\mathrm{Asn}_{66}-\mathrm{Val}_{67}$ bond was prerequisite for the maturation. Accordingly, we conclude that the processing mechanism of the GluV8 family proteases is commonly mediated by the cleavage at the $\mathrm{Asn}_{68}$ - $\mathrm{Val}_{69}$ bond of GluV8 and equivalent positions of GluSE and GluSW.

In conclusion, we established the E. coli expression system of glutamyl endopeptidases from $S$. aureus and S. warneri. This strategy may shed light on the way for the expression of the proteases that were scarcely produced in E. coli to date. 
Table 1 . GluV8-family proteases expressed in the present study

\begin{tabular}{|c|c|c|c|}
\hline No. & Protease & Description & Ref. \\
\hline 1 & GluSE & full-length form of GluSE & 14 \\
\hline 2 & GluV8 & full-length form of GluV8 & 13 \\
\hline 3 & GluSE-V8 & prepro-GluSE attached to mature GluV8 & 13 \\
\hline 4 & GluV8mut2 & $\begin{array}{l}\text { full-length GluV8 with } 2 \text { substitutions (Glu62Gln/Glu65Ser) } \\
\text { in the pro-segment }\end{array}$ & 13 \\
\hline 5 & GluV8mut4 & $\begin{array}{l}\text { full-length GluV8 with } 4 \text { substitutions (Glu62Gln/Glu65Ser } \\
\text { Ala67Pro/Asn68Ser) in the pro-segment }\end{array}$ & 13 \\
\hline 6 & GluV8mut5 & $\begin{array}{l}\text { full-length GluV8 with } 5 \text { substitutions (Asp36His/ } \\
\text { Glu62Gln/Glu65Ser/Ala67Pro/Asn68Ser) in the pro-segment }\end{array}$ & this study \\
\hline 7 & GluV8mut5 $\Delta \mathrm{C}$ & GluV8 mut5 with C-terminal 52 residues deleted & this study \\
\hline 8 & GluSE Ser235Ala & GluSE with an amino acid substitution (Ser235Ala) & 14 \\
\hline 9 & GluV8mut4 Ser237Ala & GluV8mut4 with an amino acid substitution (Ser237Ala) & 13 \\
\hline 10 & GluV8mut4 $\Delta$ C Ser237Ala & GluV8mut4 $\Delta \mathrm{C}$ with an amino acid substitution (Ser237Ala) & this study \\
\hline 11 & GluSW & full-length form of GluSW & this study \\
\hline 12 & GluV8mut5SW & prepro-GluV8mut5 attached to mature GluSW & this study \\
\hline
\end{tabular}

Nos. 1-12 correspond to lanes 1-12 of Fig. 2. 
Table 2. Relative activity of GluV8mut5 $\Delta \mathrm{C}$ toward various substrates.

\begin{tabular}{|c|c|c|c|}
\hline \multirow[t]{2}{*}{ No. } & \multirow[t]{2}{*}{ MCA peptide } & \multicolumn{2}{|c|}{ Activity (\% \pm S.D.) } \\
\hline & & - Th & $+\mathrm{Th}$ \\
\hline 1 & Z-Leu-Lue-Glu-MCA & $0.200 \pm 0.117$ & $100 \pm 6.213$ \\
\hline 2 & Suc-Ala-Glu-MCA & $0.548 \pm 0.213$ & $2.277 \pm 0.480$ \\
\hline 3 & Ac-Tyr-Val-Ala-Asp-MCA & $0.002 \pm 0.002$ & $0.727 \pm 0.008$ \\
\hline 4 & Ac-Asp-Asn-Leu-Asp-MCA & $0.000 \pm 0.001$ & $0.060 \pm 0.004$ \\
\hline 5 & Z-Ala-Ala-Asn-MCA & $0.069 \pm 0.002$ & $0.124 \pm 0.002$ \\
\hline 6 & Suc-Ala-Pro-Ala-MCA & $0.001 \pm 0.000$ & $0.002 \pm 0.000$ \\
\hline 7 & Suc-Ala-Ala-Ala-MCA & $0.002 \pm 0.002$ & $0.003 \pm 0.001$ \\
\hline 8 & Suc-Ala-Ala-Pro-Phe-MCA & $0.000 \pm 0.001$ & $0.003 \pm 0.002$ \\
\hline 9 & Z-Leu-Lue-Leu-MCA & $0.005 \pm 0.003$ & $0.004 \pm 0.002$ \\
\hline
\end{tabular}

Values are means \pm S.D. $(n=3)$. The activity of GluV8mut5 $\Delta$ C pretreated with $0.3 \mu$ g of thermolysin for Z-Leu-Lue-Glu-MCA was set to $100 \%$. The reaction mixtures were incubated at $30^{\circ} \mathrm{C}$ for $24 \mathrm{~h}$. The protein at $0.4 \mathrm{nM}$ was used for Nos. 1 and 2, whereas that at $40 \mathrm{nM}$ was used for Nos. 3-9. Data are represented as for $4 \mathrm{nM}$ proteases in all cases. 


\section{References}

[1] G.R. Drapeau, Y. Boily, J. Houmard, Purification and properties of an extracellular protease of Staphylococcus aureus, J. Biol. Chem. 247 (1972) 6720-6726.

[2] S.N. Coulter, W.R. Schwan, E.Y.W. Ng, M.H. Langhorne, H.D. Ritchie, S. Westbrock-Wadman, W.O. Hufnagle, K.R. Folger, A.S. Bayer, C.K. Stover, Staphylococcus aureus genetic loci impacting growth and survival in multiple infection environments, Mol. Microbiol. 30 (1998) 393-404.

[3] A. Karlsson, P. Saravia-Otten, K. Tegmark, E. Morfeldt, S. Arvidson, Decreased amounts of cell wall-associated protein A and fibronectin-binding proteins in Staphylococcus aureus sarA mutants due to up-regulation of extracellular proteases, Infect. Immun. 69 (2001) 4742-4748.

[4] H.R. Stennicke, K. Breddam, Glutamyl endopeptidase I. in: A.J. Barrett, N.D. Rawlings, F.F. Jr. Woessner (Eds.) Handbook of Proteolytic Enzymes, Academic Press San Diego 1998, pp243-246.

[5] C. Carmona, G.L. Gray, Nucleotide sequence of the serine protease gene of Staphylococcus aureus, strain V8, Nucleic Acids Res. 15 (1987) 6757.

[6] G.R. Drapeau, Role of a metalloprotease in activation of the precursor of staphylococcal protease, J. Bacteriol. 136 (1978) 607-613.

[7] L.N. Shaw, E. Golonka, G. Szmyd, S.J. Foster, J. Travis, J. Potempa, Cytoplasmic control of premature activation of a secreted protease zymogen: deletion of staphostatin B (SspC) in Staphylococcus aureus 8325-4 yields a profound pleiotropic phenotype, J. Bacteriol. 187 (2005) $1751-1762$.

[8] M. Sasaki, Y. Ohara-Nemoto, S. Tajika, M. Kobayashi, Y. Ikeda, M. Kaneko, T. Takagi, Purification and characterization of a glutamic acid-specific protease from Staphylococcus 
epidermidis, Jpn. J. Oral Biol. 40 (1998) 542-548.

[9] Y. Ohara-Nemoto, Y. Ikeda, M. Kobayashi, M. Sasaki, S. Tajika, S. Kimura, Characterization and molecular cloning of a glutamyl endopeptidase from Staphylococcus epidermidis, Microb. Pathog. 33 (2002) 33-41.

[10] K. Yokoi, M. Kakikawa, H. Kimoto, K. Watanabe, H. Yasukawa, A. Yamakawa, A. Taketo, K. Kodaira, Genetic and biochemical characterization of glutamyl endopeptidase of Staphylococcus warneri M, Gene 281 (2001) 115-122.

[11] M. Yabuta, N. Ochi, K. Ohsuye, Hyperproduction of a recombinant fusion protein of Staphylococcus aureus V8 protease in Escherichia coli and its processing by OmpT protease to release an active V8 protease derivative, Appl. Microbiol. Biotech. 44 (1995) 118-125.

[12] N.N. Nickerson, L. Prasad, L. Jacob, L.T. Delbaere, M.J. McGavin, Activation of the SspA serine protease zymogen of Staphylococcus aureus proceeds through unique variations of a trypsionogen-like mechanism and is dependent on both autocatalytic and metalloprotease-specific processing, J. Biol. Chem. 282 (2007) 34129-34138.

[13] T.K. Nemoto, Y. Ohara-Nemoto, T. Ono, T. Kobayakawa, Y. Shimoyama, S. Kimura, T. Takagi, Characterization of the glutamyl endopeptidase from Staphylococcus aureus expressed in Escherichia coli, FEBS J. 275 (2008) 5723-587.

[14] Y. Ohara-Nemoto, T. Ono, Y. Shimoyama, S. Kimura, T.K. Nemoto, Homologous and heterologous expression and maturation processing of extracellular glutamyl endopeptidase of Staphylococcus epidermidis, Biol. Chem. in press.

[15] Y. Ikeda, Y. Ohara-Nemoto, S. Kimura, K. Ishibashi, K. Kikuchi, PCR-based identification of Staphylococcus epidermidis targeting gseA encoding the glutamic-acid-specific protease, Can. J. 
Microbiol. 50 (2004) 493-498.

[16] T.K. Nemoto, Y. Fukuma, S. Yamada, T. Kobayakawa, T. Ono, Y. Ohara-Nemoto, The region, adjacent to the highly immunogenic site and shielded by the middle domain, is responsible for self-oligomerization/client binding of HSP90 molecular chaperone, Biochemistry 43 (2004) 7628-7636.

[17] M. Kawalec, J, Potempa, J.L, Moon, J, Travis, B.E. Murray, Molecular diversity of a putative virulence factor: Purification and characterization of isoforms of an extracellular serine glutamyl endopeptidase of Enterococcus faecalis with different enzymatic activities. J. Bacteriol. 187 (2005) 266-275.

[18] K. Breddam, M. Mendel, Substrate preferences of glutamic-acid-specific endopeptidases assessed by synthetic peptide substrates based on intramolecular fluorescence quenching, Eur. J. Biochem. 206 (1992) 103-107.

[19] E. Subbian, Y. Yabuta, U.P. Shinde, Folding pathway mediated by an intramolecular chaperon: intrinsically unstructured propeptide modulates stochastic activation of subtilisin, J. Mol. Biol. 347 (2005) 367-383.

[20] L. Prasad, Y. Leduc, K. Hayakawa, L.T. Delbaere, The structure of a universally employed enzyme: V8 protease from Staphylococcus aureus, Acta Cryst. 60 (2004) 256-259. 


\section{Figure legends}

Fig. 1. Comparison of the amino acid sequences among 3 glutamyl endopeptidases. (a) The preprosequences of GluSE [9], GluV8 [5], GluSW [10], and GluV8mut5 are aligned. Hyphens are deletions introduced for maximal matching. Amino acid numbers on the top are those for GluV8. The border between of pre- and prosequences and that between pro- and mature sequences of GluV8 and GluSE are indicated by arrows. $\quad \mathrm{X}$ indicates the proposed pro-mature border of GluSW [10]. The amino acids, Glu and Asp, which could be auto-proteolysed by glutamyl endopeptidases are underlined. Asterisks indicate the 5 amino acids substituted in GluV8mut5. (b) Amino acid identity of pre-, pro-, and mature sequences among GluSE, GluV8, and GluSW.

Fig. 2. SDS-PAGE of GluSE, GluV8, GluSW, and their derivatives. (a) The lysates (5 $\mu$ l) expressing recombinant proteins were separated by SDS-PAGE and stained with CBB (upper panel) or subjected to the immunoblotting with an anti-penta His mAb (lower panel). Arrowheads indicate recombinant proteins recognized in the lysates. (b) Aliquots of the affinity-purified proteins (5 $\mu$ l) prepared as described in "Material and methods" were separated on the SDS-PAGE gel (lower panel). Bands of purified proteins were detected and their density was quantified (upper panel). The amount of 40-44-kDa bands of GluV8 (lane 2) refers to 1. The apparent molecular masses of the major products are shown on the left. Lane 1, GluSE; lane 2, GluV8; lane 3, GluSE-V8; lane 4, GluV8mut2; lane 5,

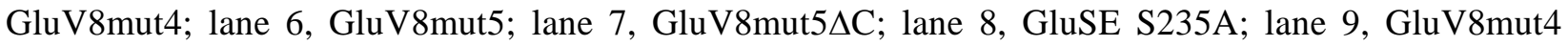
S237A; lane 10, GluV8mut4AC S237A; lane 11, GluSW; lane 12, GluV8mut5-SW. Lane M, low-molecular-weight markers. 
Fig. 3. Purification of recombinant proteases by Talon affinity chromatography. (a) The bacterial lysate from an 800-ml culture expressing the full-length form of GluV8 (open circles) or GluV8mut5 $\Delta \mathrm{C}$ (closed circles) was separated on a Talon affinity resin as described in "Materials and methods". One-ml fractions were collected, and the absorbance at $280 \mathrm{~nm}$ was determined. Inserts: Aliquots (5 $\mu \mathrm{l})$ of the fractions around the peak were separated by SDS-PAGE. Bacterial lysates and marker proteins were run in the left and right lanes, respectively. (b) Bacterial lysate expressing GluSW (open circles) or GluV8mut5-SW (closed circles) was separated and analyzed as in panel “a”.

Fig. 4. Thermolysin processing of the recombinant proteases. Recombinant GluSE (SE), GluV8mut5 (V8), GluV8mut5 $\Delta \mathrm{C}(\mathrm{V} 8 \Delta \mathrm{C})$ or GluV8mut5-SW (SW) $(3 \mu \mathrm{M})$ was incubated at $0^{\circ} \mathrm{C}$ without protease (lane 1) or processed at $37^{\circ} \mathrm{C}$ with 2.8 pmol (lane 2) or 8.4 pmol (lane 3) of thermolysin, as described in "Materials and methods". Molar ratios of recombinant proteins/thermolysin were $1 / 110$ and $1 / 37$, respectively. As a control, thermolysin (2.8 or 8.4 pmol, referred to as C1 and C2, respectively, in panels “b”-“d”) was incubated in the absence of recombinant proteins. (a) Aliquots $(0.8 \mu \mathrm{g})$ of thermolysin-untreated (lane 1) and thermolysin-treated (lanes 2 and 3) samples were separated by SDS-PAGE. Lane M, low-molecular-weight markers. (b-d) The proteolytic activities of proteins (40 nM) toward Z-Leu-Leu-Glu-MCA (b and c) and (d) azocasein were determined as described in "Materials and methods". Panel "c" is the "close up" of panel "b" concerning GluSE (SE) and GluSW (SW) Values are means \pm S.D. (n=3).

Fig. 5. Thermolysin treatment of recombinant GluSW. Recombinant GluSW (lanes 1 and 2) or 
GluV8mut5-SW (lanes 3 and 4) was incubated at $0^{\circ} \mathrm{C}$ without thermolysin (lanes 1 and 3 ) or at $37^{\circ} \mathrm{C}$ with 8.4 pmol of thermolysin (lanes 2 and 4) as described in "Materials and methods". Lane M, low-molecular-weight markers. (a) Aliquots $(0.8 \mu \mathrm{g})$ of proteins were separated by SDS-PAGE. (b) The proteolytic activity toward Z-Leu-Leu-Glu-MCA was determined. Columns 1-4 are identical to lanes 1-4, respectively, of panel “a”. Values are means \pm S.D. $(n=3)$. 
Figure 1

a
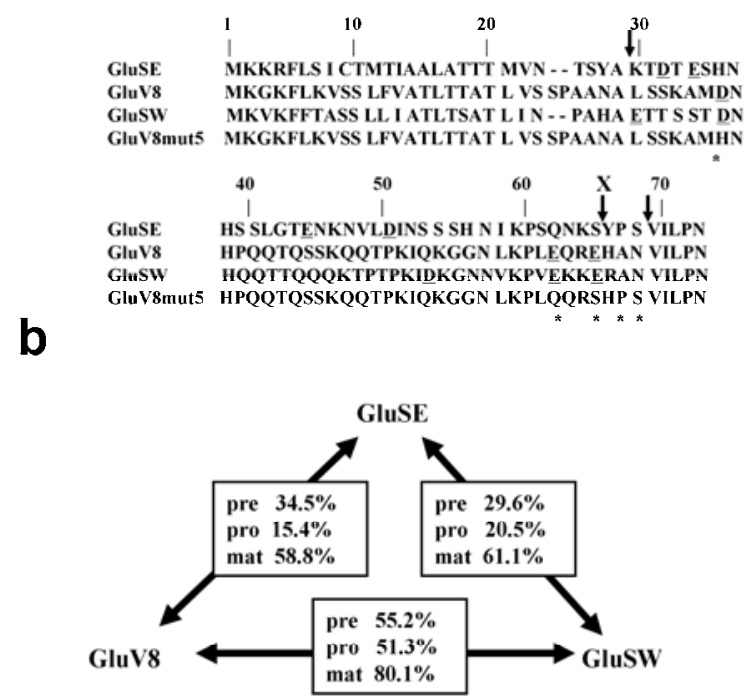

Figure 2

a

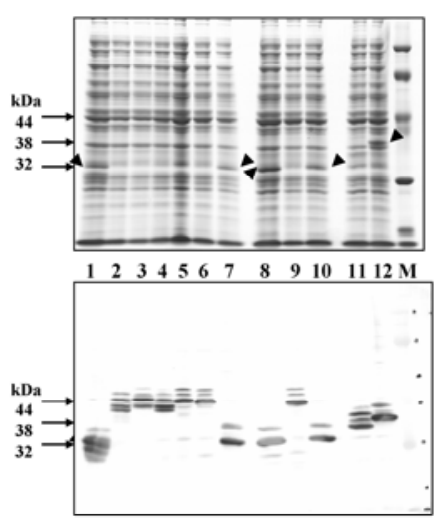

b

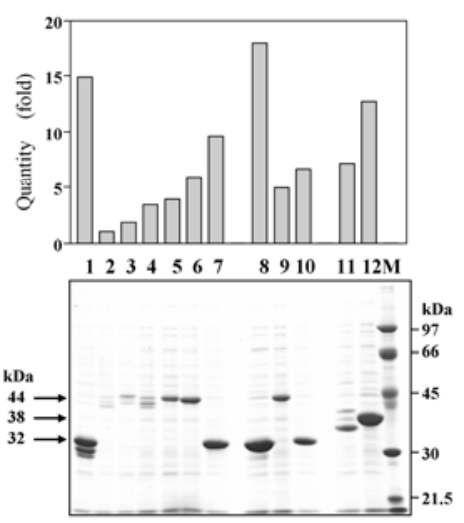


Figure 3

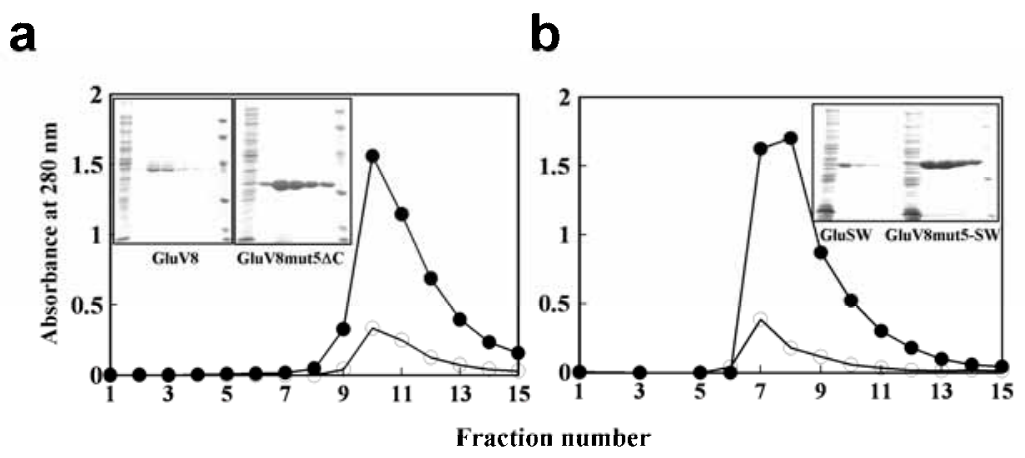

Figure 4

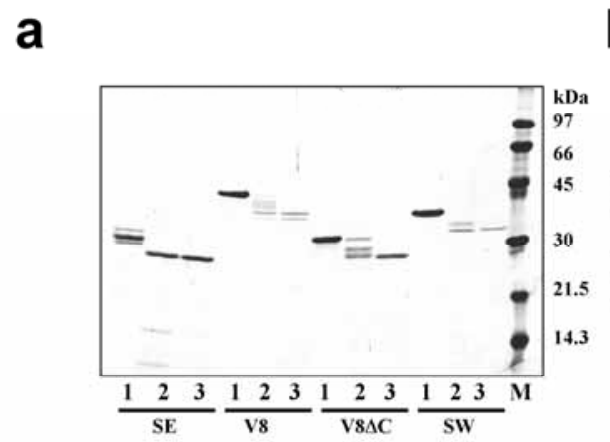

b

C
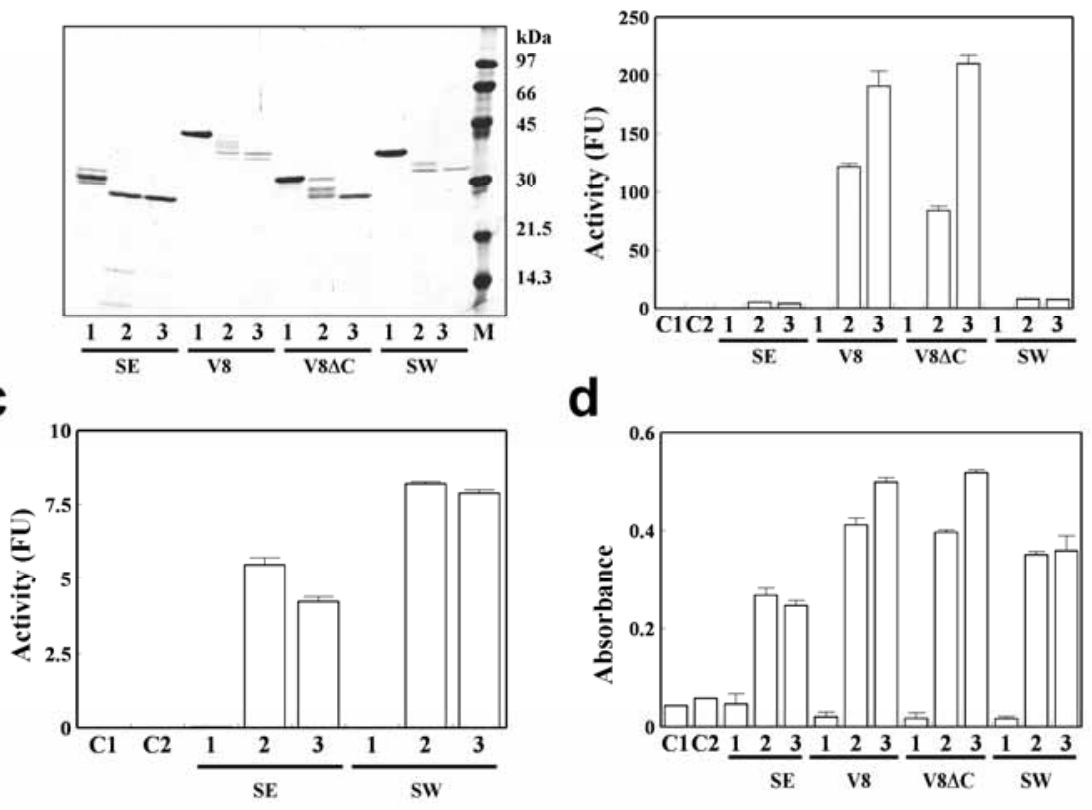

d

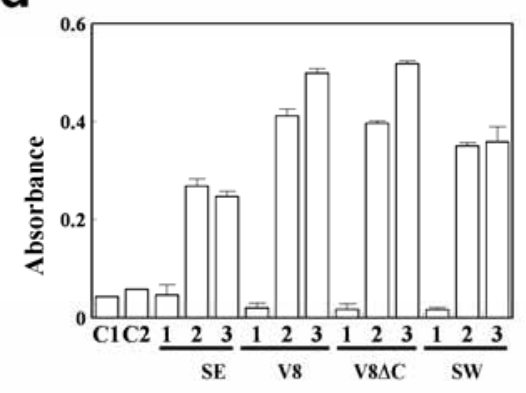


Figure 5

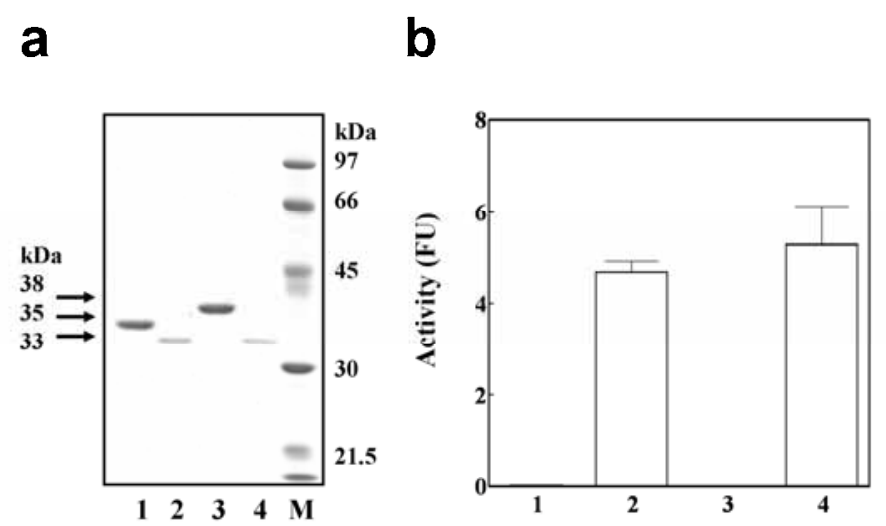

